\title{
DÉBAT. LE LOCAL, LE TERRITOIRE ET LA « PLANIFICATION ASCENDANTE »
}

\author{
Belin | « L’Espace géographique »
}

2002/1 tome 31 | pages 37 à 48

ISSN 0046-2497

ISBN 270113126X

Article disponible en ligne à l'adresse :

https://www.cairn.info/revue-espace-geographique-2002-1-page-37.htm

Distribution électronique Cairn.info pour Belin.

(C) Belin. Tous droits réservés pour tous pays.

La reproduction ou représentation de cet article, notamment par photocopie, n'est autorisée que dans les limites des conditions générales d'utilisation du site ou, le cas échéant, des conditions générales de la licence souscrite par votre établissement. Toute autre reproduction ou représentation, en tout ou partie, sous quelque forme et de quelque manière que ce soit, est interdite sauf accord préalable et écrit de l'éditeur, en dehors des cas prévus par la législation en vigueur en France. Il est précisé que son stockage dans une base de données est également interdit. 


\section{Débat}

\section{Le local, le territoire et la «planification ascendante »}

1. Ont participé au débat : Benoît Antheaume, Patrick d'Aquino, Roger Brunet, Jean-Paul Deler, Olivier Dollfus, Jean-Paul Ferrier, Gilles Lepesant, Philippe Pelletier, Thérèse SaintJulien.
Le comité de rédaction de L'Espace géographique avait jugé utile d'organiser un débat ${ }^{1}$ sur la proposition d'article qu'il avait reçue de la part de Patrick d'Aquino, compte tenu de l'intérêt des analyses et des propositions de l'auteur, et afin d'en éclairer certains aspects. Patrick d'Aquino a bien voulu se prêter à l'exercice, ce dont nous le remercions. La rédaction finale de son propre article a pu être revue à la suite de ce débat, dont nous publions ici les principaux extraits, également revus par les différents interlocuteurs.

\section{Roger Brunet}

Plusieurs d'entre nous ont souhaité débattre avec Patrick d'Aquino de quelques aspects de ses propositions. Sur un fond d'inquiétude, tant à l'égard des dangers du localisme et du communautarisme d'un côté, que du poids des autorités établies, apparentes ou occultes, de l'autre, quatre thèmes surtout nous ont retenus: celui de la définition et de l'organisation d'une pratique réellement démocratique de la "planification territoriale "; celui d'une solidarité territoriale, qui résulterait de la proximité géographique des acteurs, outrepassant quelque peu les différences sociales et culturelles; celui du rôle de l'expert sur le terrain, surtout s'il est géographe; celui de la conception même d'une planification "ascendante " et donc "transcalaire ", qui pose toute la question de l'emboîtement ou de l'autonomie des territoires, de la multiterritorialité, etc., que vous abordez à plusieurs reprises.

\section{Patrick d'Aquino}

Le terme démocratie "locale» employé dans mon texte n'implique pas une échelle particulière. Il implique le recours à la société civile à quelque échelon géographique que ce soit. Mon expérience au Sénégal repose sur 3 échelles différentes, du local $\left(3500 \mathrm{~km}^{2}\right)$ au régional $\left(20000 \mathrm{~km}^{2}\right)$. Seul le territoire peut être un lien démocratique entre les institutions et les citoyens. Le territoire présente trois avantages: c'est un espace de débat sur scène; c'est un espace de concertation; ce qui rapproche les habitants d'un territoire est d'abord la proximité spatiale, plus que la proximité d'intérêt. J'ai pu le constater jusque dans les conflits ethniques.

Que veut dire utiliser le territoire dans ce contexte de démocratie locale? C'est parvenir à un renforcement du pouvoir local et du pouvoir politique sur le territoire vis-à-vis du savoir technique. Les SIG doivent produire des résultats pour la communication avec les acteurs, non des processus, sinon c'est le savoir technique qui prend le pouvoir. Cela 
veut dire aussi appuyer la participation des citoyens à toutes les échelles de décision sur le territoire, ce qui s'oppose au principe de la subsidiarité. Pour atteindre les deux premiers objectifs, il faut provoquer le processus d'appropriation territoriale à toutes les échelles, au lieu de le replier sur le local ou sur l'ethnie. Il y a enfin interaction entre les différents niveaux pour les choix de développement.

Quels enjeux de recherche? Renforcer le pouvoir de l'acteur vis-à-vis des expertises techniques, c'est un processus d'appui à la planification impliquant l'appropriation du territoire par la population, collectivités territoriales incluses. Cela permet de passer à des actions de développement lorsque les populations ressentent le besoin de planification. Le niveau local se rend compte, lorsqu'il veut passer à la planification, qu'il doit s'adresser aux échelles supérieures, ce qui aboutit à la prise de conscience d'une multiterritorialité. Il y a beaucoup d'études à faire sur ce concept et les conditions d'appui à ce sentiment, y compris dans le mode de fonctionnement. Il existe une interaction profonde entre les différentes échelles sur une théorie d'entre local et global, par un processus d'ascendance du "terroir » au "régional ».

Certes l'enjeu n'est pas uniquement géographique. Mais le territoire est un concept de base de la géographie; l'objectif de gestion territoriale concertée est aussi très géographique; l'outil de communication, qu'est la carte sous toutes ses formes, est un outil géographique. Les questions que l'on se pose sont notamment celles de la conception d'outils interactifs entre savoir technique et citoyen, et de l'amélioration des connaissances sur les processus d'appropriation territoriale pour pouvoir appuyer le processus. La multiterritorialité est évidente, mais il faut formaliser le processus ascendant, du local au régional.

\section{Philippe Pelletier}

Le texte de Patrick d'Aquino me pose six questions.

1. Qu'un objectif de la géographie et des géographes puisse être «la gestion concertée de l'espace à différentes échelles ", je veux bien l'admettre, mais on peut parfaitement enlever " concertée ", et je préférerais au contraire ajouter " en termes d'analyse ». En effet, à partir du moment où l'on introduit le terme " concertée " on introduit un biais politique, la concertation. On entre alors dans ce domaine que l'on a appelé il n'y a pas si longtemps la "géographie active ", avec toutes ses ambiguités. Certes, tous les thèmes soulevés sont géographiques, mais dans la mesure où d'emblée on introduit cette dimension politique et idéologique on se demande: comment ça marche, qui prend les décisions, comment les prend-on?

2. Le postulat initial, "défendre les valeurs démocratiques ", est flou; certes tous ne défendent pas les valeurs démocratiques, mais beaucoup les défendent, sans être sûrs de parler de la même chose. Il y a là un débat à soi seul. Ce qui m'a gêné dans cet article est la faible part faite à l'économie. Je sais bien qu'il y a une dialectique entre le politique et l'économique, mais l'on doit se demander qui prend les décisions économiques, comment est pris en compte le marché, etc. D'une certaine façon, on pourrait aussi parler de démocratie économique.

3. J'ai pu craindre à vous lire de me trouver devant un nouveau plaidoyer pour le small is beautiful. Que ce qui rapproche les gens soit la proximité spatiale et non une proximité d'intérêts fait débat, pour le moins. Qu'entraîne réellement la proximité spatiale, et inversement l'éloignement? Les intérêts peuvent être à la fois locaux, régionaux ou entre les deux, y compris chez un individu ou un groupe d'individus. Je m'interroge sur cette multiterritorialité, je dirais plutôt d'ailleurs multispatialité. Votre expérience du Sénégal semblerait vous faire privilégier les sédentaires, mais où sont les nomades si l'on reprend cette ancienne dichotomie? Or les sédentaires eux-mêmes dans nos sociétés contemporaines sont de plus en plus mobiles, et la mobilité spatiale introduit un certain nombre de problèmes nouveaux; la mobilité des sociétés dans le domaine culturel se fait aussi de plus en plus à différentes échelles: je ne suis pas sûr que les pratiques socioculturelles soient des pratiques territoriales, est-ce qu'elles peuvent l'être quand se multiplient les festivals, les raves, etc.? 
Certes il y a là de l'espace, mais est-ce qu'il doit y avoir du territoire? Si on les territorialise absolument, il faut les encadrer politiquement et alors se pose la question de l'expression culturelle, de la liberté, etc.

4. Je ne trouve pas claire la critique de la subsidiarité, qui oblige à se référer au fédéralisme.

5. Cette réflexion sur les processus ascendants est-elle valable en tous lieux et en tous temps? Au Japon par exemple, cela fonctionne différemment me semble-t-il. Il y a des groupes de quartiers, dont on est automatiquement membre et qui gèrent le local. Il y a même une renaissance de ces pratiques dans l'ère du mégalopolisme.

6. Cette idée d'ascendance me semble bien théorique et manque un peu de chair; que donne-t-elle dans l'application?

\section{Jean-Paul Ferrier}

Comment fait-on pour que les savoirs des populations et les connaissances des experts communiquent vraiment? C'est un problème majeur dans les sociétés contemporaines. Je pense que la communication des savoirs est très insuffisante, et qu'il faut aussi qu'il y ait participation à la décision. De plus, si l'on ne décide pas en commun et que l'on ne voit pas ensuite de réalisations, échanger des idées ne sert à rien, mais crée au contraire des frustrations supplémentaires. Il faudrait encore des dispositifs qui garantissent la qualité et le bon achèvement des réalisations décidées (et quand nécessaire, l'évaluation de leur usage) : cela n'existe pratiquement jamais.

\section{Patrick d'Aquino}

Je n'ai pas de véritable réponse sur la place respective du politique et de l'économique. Les géographes et les agronomes que je côtoie ont pris l'option de mettre le politique avant l'économique, non dans une échelle de valeurs, mais dans un processus chronologique, en se disant que c'est en mettant en place un processus de responsabilisation sur le territoire que se produiront ensuite des actions économiques (agricoles ou autres). Il est vrai qu'ailleurs beaucoup de géographes abordent le territoire par les questions économiques. Il y a là un débat très large, que je ne peux aborder au fond faute d'arguments suffisants.

Sur la décision: en effet, ce n'est pas le savoir technique que je remets en cause, mais la façon dont les décisions sont prises. Car il arrive que l'on parvienne au même résultat par des processus très différents. Prenons l'exemple du tracé d'un canal dans la vallée du Sénégal. Au bout du processus de discussion et de décision, certes, on est arrivé au tracé même qui avait été prévu par les "experts "; mais loin d'être perdu tout ce temps a été utile: l'objectif n'était pas de remettre en cause le savoir technique, mais de faire intégrer un concept et de responsabiliser les acteurs. En tant que géographes, nous avons ici beaucoup à faire: qui forme ces experts? De plus en plus, on appelle des géographes dans la planification locale, dans le développement local. Dans les pays en développement, les géographes participent et souvent même décident du développement de leur pays. Qui les forme? Des géographes...

\section{Roger Brunet}

En fait, dans la richesse et le foisonnement de votre propos, vous demandez au géographe d'intervenir de quatre façons différentes. J'aimerais savoir lesquelles vous privilégiez - et ne me répondez pas "les quatre à la fois " parce qu'il existe entre elles des incompatibilités.

1. Le géographe peut être un chercheur qui travaille sur un espace déterminé, qui cherche à le comprendre, à l'expliquer, à en "rendre compte "; dans ce cas, comme le disait Philippe Pelletier, il n'a pas à entrer en " concertation » — si ce n'est la concertation entre scientifiques également informés et de niveau comparable. Il fait son métier, il a une 
posture de scientifique. Il n'est pas démontré que ce soit par là qu'il est le moins «socialement utile».

2. Il intervient aussi dans vos travaux et vos problématiques comme l'un de ces affreux techniciens dont vous vous défiez, et il risque d'être en position de force comme technicien. Je ne parle pas seulement de technicien des SIG, mais de technicien dans la connaissance du territoire. Il est l'expert, il devient donc dangereux. Paradoxe: vous voulez réduire le poids excessif que prennent les experts dans un certain nombre d'analyses sur lesquelles vous avez à travailler, mais vous en appelez au savoir du géographe. Ce sera aussi le cas, par exemple, pour le découpage de l'Afrique du Sud, qu'a observé Benoît Antheaume, où les géographes ont travaillé avec l'autorité de l'expert, laquelle est difficile à discuter.

3. Vous voulez en faire celui que nous aimerions tous être un jour ou l'autre, que j'appellerai le maïeuticien: l'accoucheur des projets par la concertation. C'est celui qui se place entre les acteurs et les experts. Celui qui, avec son bon sens de la pratique (dont se flattent volontiers les géographes), proche du terrain, mais ayant une certaine connaissance des débats abstraits de certains sociologues ou des approches techniques des architectes, fait " la synthèse " comme diraient certains, je préfère dire la maïeutique. Dans cette position, il est sans doute à certains égards, encore plus redoutable que les experts qu'il met en cause en ne se situant pas comme expert, mais comme "passeur".

4. Le quatrième rôle, que je rappelle pour mémoire, est tout simplement sa position de citoyen. Quand nous débattons sur la forme de la démocratie, nous en débattons en tant que citoyens, avec nos sentiments, nos représentations, notre idéologie, voire notre instinct; et pas spécialement comme géographes. La géographie peut nous avoir appris plus qu'à d'autres une certaine information sur la façon dont les choses fonctionnent en différents lieux, mais c'est tout. Il y a de tout chez les géographes, et même des antidémocrates. On n'en sait pas forcément plus qu'un botaniste, un sociologue ou un paysan sur cette question-là. Et c'est là un point un peu délicat: le sujet de la démocratie n'est pas ce que nous pouvons discuter le plus ou le mieux en tant que géographes. Nous savons simplement, comme tout un chacun, que certes les décisions imposées d'en haut ou de l'extérieur sont souvent mal reçues, mais que la démocratie radicale à la base est un leurre, parce que s'y expriment en réalité quantité d'intérêts individuels et de groupes, plus ou moins avoués ou évidents, et qu'il y a toujours dans un groupe social des personnes qui se font mieux entendre que d'autres; d'où l'intérêt, en effet, d'un compromis entre ces jeux de l'expression directe, des élus, des experts et, j'ajouterai, des administratifs. D'accord avec vous sur le danger des extrémistes des deux bords, "tout jacobin " ou " tout basiste ». Mais sur ces sujets, c'est une personne (parmi d'autres) qui s'exprime, sa culture géographique éventuelle n'est qu'un petit élément de sa personnalité, qui ne peut être mis en avant en tant que tel.

\section{Jean-Paul Ferrier}

Je postule qu'il y a une culture géographique qui est une grande part de la culture humaine, que cette culture géographique peut éventuellement être structurée avec des pôles de savoirs, des champs selon la formule de Roger Brunet, qu'éventuellement on peut y trouver des règles; que dans le savoir commun, dans l'expérience de l'humanité, il y a un savoir géographique qui appartient à tout le monde, qui est produit par la société et sur lequel s'exerce notre activité scientifique. Dans les territoires, il y a des hommes, qui ont un savoir immémorial, il y a là quelque chose qui est de l'ordre de ce que j'appelle le "contrat géographique ". Il y a un savoir universaliste, mais il doit servir à produire une "habitabilité " des territoires. Je crois qu'il y a une science politique à produire à l'intérieur même de notre savoir savant. En ce sens l'article de d'Aquino me semble fournir une contribution à la construction de cette théorie de géographie politique. On peut être en désaccord avec cette position, ce n'est pas grave, le tout est 
de débattre. Moi j'aurais tendance à être d'accord avec ce que j'ai compris de sa position sur la politique. Pour moi, un savoir géographique est nécessairement appliqué, parce que dans ce savoir géographique, que je considère comme une part de la culture humaine, il y a forcément de la politique.

\section{Philippe Pelletier}

Tu sous-entends que la science géographique, une " géopolitique » bien comprise, donne un projet social par exemple, ou un projet d'habitabilité? C'est une chose; autre chose est la sensibilité à ces questions-là: je me retrouve tout à fait dans les quatre points qu'a évoqués Roger Brunet. Il y a toujours de grands risques dans le passage de l'un à l'autre, à partir du moment où d'une sensibilité on prétend passer à un encadrement strict: la Geopolitik des années 1930 en a fourni un exemple caricatural.

\section{Jean-Paul Ferrier}

Je comprends que l'on puisse refuser cette démarche, mais je pense que dans le savoir géographique il y a forcément un savoir politique à expliciter, nécessairement, puisque dans chaque "place " il y a de la concurrence, du conflit, des règles pour gérer les conflits, voire la guerre.

\section{Philippe Pelletier}

Il y a des préoccupations sociales, économiques ou culturelles, mais il n'y a pas forcément de conflit; et si oui, est-ce qu'il y a un modèle géographique de gestion du conflit?

\section{Jean-Paul Ferrier}

Pas encore, et pourtant il faut instaurer une "pacificité » des territoires.

\section{Philippe Pelletier}

D'Aquino l'a écrit dans son texte: la politique c'est la bonne gestion du conflit.

\section{Patrick d'Aquino}

Je reprends les points qu'a soulevés Roger Brunet. On fait trois métiers différents : la même personne ne peut pas faire les trois métiers, mais on peut les trouver chez les géographes. Le premier, le scientifique, et le deuxième, l'expert, pas de problème. Le troisième, effectivement, je considère qu'aujourd'hui on en a besoin. Est-ce un besoin de géographe? Je ne vois pas qui d'autre pourrait agir sur cette interaction là, sur le territoire; je ne vois pas quelle autre discipline peut être utilisée; c'est pour cela que je fais de la géographie. C'est là que l'on peut le mieux aborder ces problèmes, où il $\mathrm{y}$ a effectivement des interactions entre des politiques et des usages des territoires. C'est vrai qu'il y a un énorme risque parce qu'on se retrouve sur un terrain flou, où les sentiments peuvent prendre le pas sur la méthodologie; c'est pour cela que dans mon article j'ai dit beaucoup de choses sur la démocratie et sur le politique: parce qu'effectivement c'est l'un des trois métiers où, vu les risques de dérives politiques ou citoyennes, il me semble essentiel d'expliciter le mieux possible quels sont les choix citoyens et politiques qui se cachent: je rejoins Jean-Paul Ferrier, car même si on est un expert sur le territoire, on a des choix non explicités. Et dans ce troisième métier, qui est beaucoup plus délicat et plus risqué, l'une des façons d'éliminer le risque est d'expliciter les choix cachés. Et là on sort de la géographie.

\section{Roger Brunet}

Je vais poursuivre mon petit travail de maïeuticien local: ce qui me parait résulter de ce que vous venez de dire, c'est que le géographe n'est légitimé pour faire tout ce que vous lui assignez que parce qu'il est le moins mal placé pour parler de territoire. C'est d'accord, 
je partage entièrement ce point de vue et je l'ai déjà écrit ou dit à maintes reprises. Par rapport aux deux éléments que soulignait Ferrier tout à l'heure, la démocratie d'un côté, science et action de l'autre, l'important ce n'est ni le premier, ni le second, mais le troisième, celui qu'il n'a pas dit: c'est-à-dire notre réflexion sur le territoire; et c'est pour cela que je vous ai posé des questions sur la façon dont vous pensiez ces territoires, sur l'emboîtement, la multiterritorialité, etc. Je crois que c'est là que nous avons le plus de progrès à faire, parce que la littérature sur la forme de la démocratie, vous l'avez rappelé vous-même dans votre article, est considérable et bien établie; la littérature sur science et société est, elle, surabondante et nous n'y apporterons pas énormément de choses originales - des témoignages locaux intéressants, oui, mais il n'est pas sûr que nous ayons là-dessus des révélations sensationnelles à faire. En revanche, sur ce qui est notre lieu d'action, qui est le territoire, la territorialisation, nous ne sommes pas les seuls bien entendu, mais nous sommes sans doute les moins mal placés et c'est là-dessus que je souhaitais que votre article soit un peu plus clair. On retrouve ici d'anciens débats trentenaires sur l'autogestion, sauf que ces débats portaient très peu sur le territoire; même si le terme d'autogestion est maintenant considéré comme une vieille lune, quelle est la part que vous faites jouer aux intérêts des acteurs locaux, exprimés dans leur spontanéité ou cachés dans leur roublardise, et qui ne peuvent jamais être tous également satisfaits?

Il me semble que le voisinage, la proximité, en effet, peuvent créer des solidarités de fait; mais n'est-ce pas en fonction d'intérêts personnels (ou de groupe) bien compris, qui localement et momentanément transcendent les autres: face à un risque d'inondation, un besoin de sécurité, aux avantages ou aux nuisances de nouvelles implantations, etc.? Et attention à l'échelle: on sait aussi que tout projet d'aménagement local entraîne de nouveaux clivages, dont certains sont strictement territoriaux; ceux qui sont proches d'un nouvel équipement n'ont pas les mêmes intérêts que ceux qui en sont loin, ou très loin (ravis ou malheureux, dans un sens ou dans l'autre selon les cas). C'est là une manifestation des fameuses "rentes différentielles " dans l'espace géographique, ce qui est à la fois géographique, politique et économique. Je souhaite que vos travaux et votre expérience nous en disent davantage sur ces sujets.

\section{Philippe Pelletier}

Cette problématique d'autogestion, qui passe de l'économique au territoire, elle me semble portée surtout dans le monde rural, alors que dans les milieux urbains on assiste à quelque chose d'un petit peu différent, qui part dans tous les sens; or le problème est que l'on se trouve dans un monde de plus en plus urbanisé.

\section{Roger Brunet}

Toutefois les réactions différentielles face à des projets se produisent aussi en ville, et de plus en plus avec les nouveaux tracés de tramways, les complexes commerciaux ou de loisirs, la perception des risques industriels, le retranchement de certains quartiers, etc.; et il me semble que les groupes d'action municipale (GAM) dont Dubedout fut un pionnier n'étaient pas très différents?

\section{Philippe Pelletier}

Néanmoins j'ai l'impression que cette réflexion de géographes " citoyens » ou " maïeuticiens » doit fonctionner mieux dans des espaces ruraux ou néoruraux que dans des mégapoles, en tous cas d'un point de vue pratique, si l'on pense à obtenir un peu de succès car si l'on échoue c'est très frustrant. C'est peut-être un cliché, mais on retombe ici sur la question du voisinage: qui connait en ville son voisin? qui a envie de partager ou de faire quelque chose avec son voisin dans le quartier? Certes beaucoup, y compris les partis politiques et les associations, parlent de renouveau des quartiers, etc.; mais je m'interroge. 


\section{Olivier Dollfus}

Certaines ruelles de mégapoles comme Tokyo peuvent être assimilées à des villages de ce point de vue.

\section{Thérèse Saint-Julien}

Il est vrai que l'autogestion ne s'occupait pas de territoire, sauf un peu dans l'expérience des GAM effectivement. Mais il me semble que notre débat est traversé par un autre débat, qui est celui du développement économique local, auquel participent beaucoup d'économistes. C'est la question posée par les districts industriels italiens et Michael Storper, qui est géographe, a beaucoup écrit sur cette question de voisinage, de proximité. Dans ces analyses, c'est la démocratie participative qui est censée faire émerger le projet économique. Il y a là comme une lame de fond, qui dépasse l'enjeu de la mise en pratique de l'aménagement du territoire au sens propre. Sans doute, la production de littérature géographique des vingt dernières années a été plus abondante sur ces questions-là que sur celle que vous abordez, qui est celle de la participation locale, de la participation dans l'élaboration du projet territorial. Quand Storper parle des conventions qui sont possibles dans le voisinage, quand on analyse la structuration des réseaux familiaux, c'est de la démocratie participative; mais on ne parle pas des mêmes acteurs: il s'agit de décisions de firmes. Il y a là quelque chose qui se rattache à ce que vous dites, à des hypothèses qu'on fait sur le territoire.

\section{Jean-Paul Ferrier}

Cette construction politique, ou cette approche territoriale avec une notion de gouvernance, qui est pensée à partir du Sénégal, est parfaitement imaginable partout dans le monde. Je l'ai parfaitement ressenti pour la France. Vous avez donné l'exemple du Japon avec l'obligation d'appartenir à des sortes de quartiers, une sorte de résurgence du système féodal, ou l'exemple des petites rues. La question est: avons-nous besoin d'avoir des relations avec des voisins? Dans les sociétés dans lesquelles nous sommes entrés, que je considère comme métropolisées et dans lesquelles la question du rural ne se pose pas, il peut $\mathrm{y}$ avoir des comportements de style rural. Le texte de d'Aquino propose quelque chose, il ne rejette rien de ce qui existe et il essaie de faire fonctionner cette sorte d'interterritorialité qui prend appui sur la proximité spatiale. Il y a un jeu qui n'est pas de l'ordre du réseau, qui est de l'ordre de la proximité, ce qui renvoie aux discussions sur l'opposition territoireréseau. Il n'y a pas de territoire sans réseaux et il n'y a pas de réseau sans territoire: le problème est de savoir comment cela s'articule à un moment qui est le nôtre. Je pense que ce texte propose une voie cognitive, pragmatique: si on le suivait, les choses se passeraient peut-être un peu différemment. Que pensons-nous de cette problématique? est-ce qu'on la considère comme une grande question? Je pense que si l'on ne gère pas autrement les questions de proximité, si nous ne savons pas penser la multiproximité, nous ne pourrons pas limiter la violence terrible et aveugle que n'importe quel voisin peut provoquer.

\section{Benoît Antheaume}

D'Aquino a toujours parlé de proximité spatiale, toi tu parles d'autre chose. Par exemple, si d'Afrique du Sud j'envoie un message électronique à la rédaction de L'Espace géographique, j'ai une réponse dans l'heure, c'est une forme de proximité qui n'est pas du tout spatiale, mais qui est incontestablement une proximité. Je voudrais revenir sur les voisins, j'habite dans un pays où l'on crée du voisinage. Le Goix a écrit un article dans l'Espace géographique sur les gated communities, où il montre qu'à partir du moment où l'on ferme un quartier, on crée du territoire, on crée des voisins, dès lors qu'on entre et qu'on sort par une seule porte. Tu as forcément des voisins, que tu ne choisis pas. Quand autrefois on créait les townships et que l'on assignait les populations à résidence sur un territoire en fonction de critères qui étaient évidemment tout à fait 
détestables, on créait aussi des voisinages. Je ne sais pas si cette proximité spatiale obligée et obligatoire est un bon départ pour créer du «local».

\section{Roger Brunet}

D'Aquino fait le pari que c'est un bon départ pour essayer de traiter les conflits d'intérêt. C'est une hypothèse forte.

\section{Philippe Pelletier}

Est-ce que cela peut s'appliquer dans la ville actuelle? j'en doute.

\section{Patrick d'Aquino}

Il faut bien comprendre ce que j'ai dit: le voisinage n'est pas une entrée en soi, c'est un fait.

\section{Gilles Lepesant}

La question des pouvoirs locaux connaît une nouvelle actualité : au niveau européen, vient d'être publié un "livre blanc " sur la gouvernance et les débats sur l'Europe consacrent une large place à la notion de subsidiarité. La rédaction de la Charte des droits fondamentaux adoptée au Conseil européen de Nice a donné lieu à un débat impliquant plusieurs niveaux de pouvoir en Europe sur l'opportunité d'invoquer un " héritage religieux " propre à l'Europe. Le Land de Bavière, relayé par le Président allemand de la Convention (R. Herzog) a tenté d'imposer une mention de cet héritage, refusée par l'exécutif français. Quand d'Aquino pose la question de la relation entre espace et pouvoir, ce n'est pas seulement de développement local ou de démocratie locale qu'il s'agit, mais plus largement des nouvelles configurations, de nouveaux jeux entre les différents niveaux de pouvoir territoriaux, avec de nouvelles formes de production de compromis (la Convention dans le cas précis). À l'échelle européenne, il me semble que ce qui est en jeu, c'est la remise en cause, fondée ou non, du cadre étatique comme cadre exclusif du débat démocratique et comme cadre principal d'action. De là découle une série de questions sur la légitimité des nouveaux acteurs qui apparaissent, sur l'articulation entre les différents niveaux de territorialité, sur la référence des sociétés à plusieurs territoires, autant de questions dont les géographes sont fondés à débattre. Pour citer le cas de l'Europe centrale, une articulation nouvelle apparaît, parfois génératrice de tensions, entre les territoires historiques relevant de configurations territoriales périmées mais pas toujours oubliées, les territoires étatiques et nationaux, le territoire institutionnel de l'Union européenne, etc. Dans ce cas spécifique, l'enjeu des pouvoirs locaux ne relève pas du seul développement local. Ce qui est en cause est beaucoup plus vaste et plus important. Peut-être peut-on en rendre compte en analysant les liens qui régissent les rapports entre l'acteur, l'espace et le temps.

\section{Roger Brunet}

D’Aquino le rappelle au début de son article: un certain nombre de militants de la démocratie locale à la base sont, d'une certaine façon, de ceux qui considèrent qu'il ne faut pas d'autorité étatique et que les conflits se résoudront par l'initiative, l'entreprise, etc.; c'est un avatar, une forme particulière de ce que d'autres appelleraient la mondialisation, et ce n'est pas nécessairement "progressiste " si cela renvoie, de fait, à une manifestation de la loi du plus fort dans les assemblées locales. Le débat existe aussi à des niveaux intermédiaires: certains plaident pour une "Europe des régions »; il m'est arrivé de m'exprimer négativement sur ce sujet, où je redoute plus de dangers que de progrès parce que vois faire les caciques locaux, les jeux de pouvoirs à l'échelle régionale, qui sont au moins aussi redoutables qu'à l'échelle nationale: on n'a pas forcément très envie de voir se développer la Ligue Lombarde, le Vlaams Blok, ni même les pouvoirs de certains présidents de nos conseils généraux ou régionaux. 


\section{Gilles Lepesant}

À propos de l'héritage religieux que j'évoquais, l'initiative venait en effet d'une région, la Bavière, qui avait réussi à faire passer son message, créant un conflit majeur sur une simple phrase porteuse de sens. On est là dans un jeu qui laisse de côté tous les cadres auxquels on est habitué, notamment le cadre étatique, et qui crée des relations nouvelles entre les acteurs au niveau européen.

\section{Patrick d'Aquino}

Le but n'est pas de remettre en cause le cadre étatique, c'est de remettre en cause le cadre d'un seul niveau territorial. Ce n'est pas l'Europe des régions, des États, etc.

\section{Roger Brunet}

Toutefois même à l'échelon auquel vous vous placez, en mettant en cause par exemple la domination locale des experts sur les projets d'aménagement, vous pouvez aussi parfaitement viser par là le cadre étatique parce que, souvent, ces experts sont précisément les représentants de l'État, ou considérés comme tels. La parade classique est d'ailleurs de parvenir à faire adopter la position de l'expert comme position spontanée de la population locale. C'est une tactique bien connue. Or l'État n'est pas un étrange ennemi envahissant, c'est nous, à travers nos élus. Bien sûr, ses représentants peuvent être mauvais; les notables locaux aussi; voire le chercheur impliqué...

\section{Patrick d'Aquino}

On ne fait pas de planification, on a engagé les choses de telle sorte que l'on s'est dit que l'on poserait la question de la planification lorsque le besoin s'en ferait sentir localement. Le processus a démarré dans la responsabilisation à outrance des acteurs et des collectivités locales, c'est-à-dire un jeu d'animation institutionnel pour laisser réellement, sur les territoires sur lesquels on travaille, carte blanche aux acteurs et aux autorités locales; les laisser prendre les décisions par l'apprentissage. Le problème de la première étape était que tous les acteurs prennent conscience de cette responsabilité nouvelle, qui n'a rien à voir avec la décentralisation qui, là-bas, n'a pas de sens. Cela veut dire aussi travailler et harceler les experts, les choisir par rapport à leur approche sur les questions qui intéressent les gens, cela implique pour les experts de se mettre totalement en retrait au départ. On laisse les gens agir et la première chose qui apparait c'est que l'on discute de la réglementation collective. Avoir un résultat de qualité : c'est quand le processus de discussion et d'élaboration a été de qualité, que les gens sont contents. De toute façon, il n'y a pas de vérité absolue face à un enjeu complexe; nous n'avons pas l'impression que le savoir technique est forcément infaillible. C'est toujours une solution imparfaite mais qui s'enrichit au fur et à mesure. La planification est vue comme cela.

Deuxième étape, après ces règles collectives, cette réorganisation entre eux de l'usage du territoire, quand ils s'aperçoivent que cela ne suffit pas, on passe à un réaménagement physique par exemple. Ce n'est pas encore de la planification parce qu'il n'y a pas de questionnement sur ce que l'on veut faire dans dix ans, c'est une action localisée sur le territoire où l'expert a en face de lui des gens qui maîtrisent mieux leur pouvoir, qui sont conscients de leur pouvoir, qui sont capables de lui dire non. C'est là que des géographes apparaissent comme des experts.

La troisième étape, c'est quand ils arrivent à se rendre compte que ce n'est pas en faisant un pont là, une mare ici ou une nouvelle école ailleurs que tout va mieux. Des incohérences apparaissent entre les projets locaux. Il peut y avoir une cohérence locale qui devient une incohérence à l'échelon supérieur: alors apparaît le besoin de discuter avec les voisins et avec le niveau supérieur et c'est ainsi que l'on rentre dans la planification proprement dite. Ce qui m'intéresse est de savoir avec quels outils, quels supports 
méthodologiques, quelles cartes, quelles données on peut appuyer ce processus, on peut l'enrichir. Quand on a des problèmes complexes de territoire, ce n'est pas seulement d'une carte dont on a besoin: il ne faut pas nier la complexité parce que l'on travaille avec des acteurs, le défi a été de leur apprendre à concevoir une organisation de l'information, à sélectionner des informations, à être capables de sentir la limite de l'information, la limite de la carte qui sort du SIG par exemple; savoir qu'en fait ce ne sont pas des résultats parfaits, qu'il y a des imperfections, leur montrer que s'ils construisent leur propre système, ils connaîtront bien, ils sentiront bien de façon implicite les problèmes, ils verront bien les limites des cartes.

Des travaux intéressants ont été faits sur les raisons pour lesquelles ils n'ont pas utilisé les résultats de façon stricte, y compris les simulations. C'est la peur de la planification mais c'est en même temps un processus qui va vers la planification, qui va obligatoirement vers les autres et qui obligatoirement remonte vers la région. Et on arrive à les intéresser à ce qui se passe dans les territoires voisins parce que cela influe sur leur propre territoire. Tout ce qui est problème de l'eau, par exemple, est très bien compris comme bien collectif à partager et à planifier. Nous pensions qu'une des premières questions qui serait abordée serait la question de l'eau: pas du tout, parce que la question de l'eau dépasse le niveau strictement local; ils ne l'ont jamais abordée d'entrée. Et quand ils arrivent à l'échelle où se pose la question de l'eau, ils l'abordent. Il a été très intéressant d'utiliser les jeux de rôles et les simulations multi-agents autour des cartes dans ces processus parce que c'est tellement interactif que c'est très efficace.

\section{Gilles Lepesant}

Quels sont les « ils» dont vous parlez?

\section{Patrick d'Aquino}

Cela fait aussi partie de notre méthode: au départ, il n'était absolument pas question d'identifier des acteurs privilégiés. Dans la démarche participative, on crée un cadre de concertation. On part des élus locaux pour essayer de les mettre face à leurs responsabilités devant des choix: c'est un apprentissage. Leur dire qu'ils seront responsables des dégâts sociaux éventuels. L'expert se détache toujours des responsabilités, il est extérieur. C'est progressif: en trois ans de processus, il y encore un acteur dans cette vallée, les pêcheurs, qui n'ont pas été convoqués. Depuis le début on s'en rend compte, mais on ne dit rien, on attend, maintenant que l'on en arrive à l'eau, on va s'intéresser aux pêcheurs. Il y en a d'autres qui sont occultés, le but n'est pas que cela soit parfait, l'objectif est d'enrichir de plus en plus le processus pour que le plus de gens possible soient concernés.

\section{Benoît Antheaume}

En Afrique du Sud, il existe maintenant des chaires universitaires de savoir « indigène » pour faire pièce au savoir technique. Mais on peut remarquer que pour faire le tracé nord du TGV par les experts (entre Paris et Lyon) il a fallu trois ans, et pour faire le tracé sud (entre Lyon et Marseille), avec le "savoir indigène " en plus, il a fallu dix ans...

\section{Patrick d'Aquino}

On ne cherche absolument pas à recueillir le savoir indigène.

\section{Jean-Paul Ferrier}

Sinon quelqu'un parlera au nom du savoir indigène.

\section{Roger Brunet}

Merci pour toutes vos contributions. Il est clair que nous souhaitons que Patrick d'Aquino 
nous propose aussi une illustration originale de ses propos en présentant concrètement le travail réalisé dans la vallée du Sénégal (c'est l'objet du second article).

Débat retranscrit et adapté par Régine Vanduick et Roger Brunet, revu par les interlocuteurs.

\section{Complément au débat, par Patrick d'Aquino}

Notre approche du territoire se distingue par sa dimension symbolique, institutionnelle et politique, voire idéologique (c'est-à-dire une certaine définition de la démocratie). Nous sommes donc à l'opposé d'une approche technico-économiste définissant le territoire comme l'espace d'une activité ou d'une dynamique particulière. Implicitement, le territoire a souvent été simplement intégré en tant que base spatiale (Passet, 1979; Thireau, 1993), "systémique ", des phénomènes socio-économiques du développement local (Pecqueur, 1989; Courlet, 1994; Calame, 1994; Veltz, 1997), qu'ils s'appellent systèmes productifs locaux (Coujard, 1982; Huttin, 1982), systèmes agraires (Brun, 1982), formations socio-spatiales (Di Méo, 1991), etc. Le territoire est alors perçu comme un critère de densité de relations sociales et économiques, où la proximité géographique est moins significative en elle-même que comme condition de telles relations. Mais du coup, entre activités économiques et espace géographique, il semble difficile de définir un niveau territorial pertinent qui puisse superposer assez justement les limites d'un espace géographique avec celles d'un ensemble de systèmes sociaux de production (Huttin, 1982). Les tenants de la modélisation systémique des activités agraires ${ }^{2}$ ont évacué le problème en définissant a priori des échelles géographiques (exploitation, village, terroir, région, etc.), dans lesquelles ils ont ensuite appliqué l'analyse systémique.

Derrière la volonté d'aménagement du territoire, c'est l'organisation de l'espace technico-économique qui est recherchée de la sorte: en suivant les corrélations des répartitions spatiales des activités, on parviendrait ainsi à déceler des territoires. Ainsi la formation socio-spatiale de G. Di Méo, qui se particularise par sa dimension économique, constitue en quelque sorte une maille d'intervention, pour un aménagement du territoire au-dessus des communes. C'est avec une perception très proche, et à peu près en même temps, que certains agronomes ont conçu une approche articulant filière et territoire (Vissac et Benoit, 1993; Deffontaines, 1994; Pernet, 1994). Cette dernière repose sur l'hypothèse que les processus localisés de développement sont les produits d'une articulation entre des dynamiques régionales, effets obligés de tendances globales, et des particularismes locaux. C'est par conséquent une approche géographique et économique qui cherche à réaliser la jonction entre l'étude des dynamiques régionales d'intégration au global et l'étude des dynamiques locales, appréhendées par le système rural localisé (Pernet, 1994), concept issu de l'économie industrielle des systèmes productifs locaux (Benko et Lipietz, 1992). Le système rural localisé, défini à partir d'indicateurs statistiques agro-économiques et de la transcription spatiale de faits socio-économiques, est perçu comme un moyen de compréhension de la société locale, à l'interface entre dynamiques globales et capacités locales (Deffontaines, 1994).

En fin de compte, ces démarches structuralistes et systémiques sont proches de l'idéologie du développement local, en privilégiant elles aussi l'appui aux acteurs les plus dynamiques et la mobilisation économique de la société civile, se penchant ainsi surtout sur des entités spatio-économiques d'une certaine originalité et vitalité (Benko et Lipietz, 1992; Di Méo, 1998). La dimension politique, confondue avec les structures politicoadministratives et décrétée en crise, y est quelque peu évacuée. En définitive, ces approches se focalisent sur le développement technique plutôt que sur la qualité des processus de décision, ce qui entraîne une représentation ambiguë du territoire chez ces adeptes du développement local. D'un côté, le territoire apparaît comme partie essentielle
2. Formulation préférable à celle de "système agraire ", le système n'étant qu'une construction intellectuelle, un modèle parmi d'autres, d'une réalité (Landais, 1994; J. Weber, communication personnelle). 
(Pecqueur, 1989; Vachon, 1993; Calame, 1994); d'un autre, les instances qui sont chargées de le gérer et de le transformer sont négligées. Les élus locaux, les collectivités locales ou les responsables politiques coutumiers des territoires locaux sont rarement évoqués dans ces approches, si ce n'est comme l'un des partenaires périphériques du processus.

Ces approches technico-économiques ne se situent donc pas dans la même idéologie de l'action que notre propre perspective: alors qu'elles cherchent à identifier un cadre cohérent à l'intervention technique, évacuant, dans la lignée du développement local, la dimension politique, nous nous situons à l'inverse dans l'appui à une réhabilitation de la dimension sociopolitique dans toute décision sur le développement d'un territoire. Notre territoire est l'espace socialisé de toute une société locale, non celui d'une activité. L'émergence d'une identité territoriale ne peut se résumer ni à un simple "espace vécu " ni à une activité économique commune sans existence politique et socioculturelle reconnue. Si la facette économique du territoire peut sembler préférable pour l'identification de territoires avérés (Di Méo, 1998), elle n'en est pas une étape génitrice ou constitutive. L'activité économique n'est ici considérée que comme l'un des produits d'un territoire fonctionnel, contrairement à d'autres auteurs qui considèrent que la facette économique est originelle ou tout au moins essentielle. Ce type d'action de l'homme sur l'espace, et les logiques de structuration et d'organisation spatiales qui en découlent, est plutôt considéré ici comme les produits, et non les origines, de l'« idée » territoriale. Cependant, l'identité territoriale ne se réduit pas d'avantage à une identité politico-juridique ou à l'enracinement dans un lieu: il y faut un peu de tout cela et quelque chose de plus, les sentiments d'appartenance (Brunet, 1992). L'histoire de chaque identification territoriale peut par conséquent être très différente et provenir de phénomènes et de contextes très variables. C'est pourquoi, plutôt que de privilégier l'une des dimensions dans la définition du phénomène identitaire territorial ou de se hasarder dans une distribution du poids de ces différents éléments en son sein, nous préférons ici, en accord avec notre idéologie de l'action, réfléchir à un processus croissant d'émergence d'une nouvelle identité territoriale active: l'accompagnement étape par étape d'une dynamique d'appropriation territoriale, par une responsabilisation initiale sur l'espace qui aboutira in fine à son appropriation.

Vient de paraître dans la collection Atlas de France RECLUS, dirigée par Thérèse Saint-Julien

\section{TERRITOIRE ET AMÉNAGEMENT}

Coordination scientifique : Yves Guermond,

avec la collaboration d'Emmanuelle Bonerandi, Patricia Cicille et Thérèse Saint-Julien

Collaborateurs: Nicolas Archambault; Bernard Aubry; Franck Auriac ; Benjamain Brillaut; Michel Bussi; Laurent Chapelon; Philippe Chataignon ; Daniel Delahaye ; Jean-Christophe François ; Christophe Granier ; Claude Grasland ; Jean de Hédouville ; Emmanuelle Le Dorlot ; Hélène Mathian ; Alexandre Moine ; Bernard Reitel ; Isabelle Roussel ; Ėve Sastre-Conte ; Christophe Terrier ; Vincent Vallès ; Vincent Vandererven.

Table des matières:

L'aménagement du territoire; chap. 1. Un territoire en mutation; chap. 2. L'État aménageur; chap. 3. Gestion des crises, décentralisation; chap. 4. Environnement et territoire; chap. 5. L'aménagement du territoire entre Europe, État et régions; Conclusion : Entre discours et action.

2001, 144 pages, 176 cartes et graphiques.

Diffusion : La documentation Française, 124 rue Henri Barbusse, 93308 Aubervilliers, tél. : 0140157000 , fax : 0140156800 